\title{
Indoor location systems in emergency scenarios - A Survey
}

\author{
Arivan S. Bastos \\ Universidade Federal da Bahia \\ Instituto de Matemática \\ Av. Adhemar de Barros $\mathrm{s} / \mathrm{n}$ \\ Salvador, BA, Brazil \\ arivanbastos@gmail.com
}

\author{
Vaninha Vieira \\ Universidade Federal da Bahia \\ Instituto de Matemática \\ Av. Adhemar de Barros $\mathrm{s} / \mathrm{n}$ \\ Salvador, BA, Brazil \\ vaninha.vieira@gmail.com
}

\author{
Antonio L. Apolinário Jr \\ Universidade Federal da Bahia \\ Instituto de Matemática \\ Av. Adhemar de Barros $\mathrm{s} / \mathrm{n}$ \\ Salvador, BA, Brazil \\ apolinario@dcc.ufba.br
}

\begin{abstract}
Indoor location data are critical in emergency situations. Command centers need to monitor their operational forces. Rescuers need to find potential victims to carry proper care and the building's occupants need to find the way for fast evacuation. Despite the growing body of research in indoor location, no technique is considered appropriate for different situations. Furthermore, few studies have analyzed the applicability of these techniques in an emergency setting, which has particular characteristics. This survey reviews works in indoor location applied to emergency scenarios, analyzing their applicability in relation to existing requirements in these types of situations.
\end{abstract}

\section{Categories and Subject Descriptors}

C.3 [Special-purpose and application-based systems]: Real-time and embedded systems; D.2.8 [Computer-CommunicationNetworks]: Distributed Systems - client/server

\section{General Terms}

Theory

\section{Keywords}

indoor positioning, indoor navigation, emergency management

\section{INTRODUCTION}

In emergency scenarios the fast conduction of victims to exit (evacuation) and the precise monitoring of the rescuers ${ }^{1}$ position are both important to reducing deaths and injuries.

\footnotetext{
${ }^{1}$ We consider "rescuer" as any operational forces member, comprising medical, police and fire brigade.
}

Permission to make digital or hard copies of all or part of this work for personal or classroom use is granted without fee provided that copies are not made or distributed for profit or commercial advantage and that copies bear this notice and the full citation on the first page. To copy otherwise, to republish, to post on servers or to redistribute to lists, requires prior specific permission and/or a fee.

SBSI 2015, May 26th-29th, 2015, Goiânia, Goiás, Brazil

Copyright SBC 2015.
To monitor rescuers command centers use radio communication, where the team member directly reports his/her position. In situations of stress, disorientation or unconsciousness the active reporting strategy may fail [11].

Location positioning systems can be used to automatically gather the positioning of rescuers and victims. The most usual location technique, the GPS, does not work well indoor because of interferences generated by physical barriers (such as walls and furniture) and sources of radio signal [17]. Thus, many techniques have been developed to provide the location of mobile devices in indoor environments with greater accuracy. However, emergency situations have particular characteristics (e.g., smoke, blackout) that can reduce visibility and make useless the current indoor positioning systems (IPS).

Most of existing surveys approaching location systems focus on the adopted technique, without considering the specifics of emergency scenarios [35, 19, 12, 14, 32, 39]. Only one review of indoor positioning systems for emergency situations was identified [6]. However, it is from 2011 and does not connect the characteristics of emergencies scenarios with most modern techniques.

Besides, there is a lack to categorize the requirements of emergency situations for indoor location systems. For example, luminosity is essential for indoor location techniques based on images. However, on emergency situation we can experiment scenarios as lack of power or presence of smoke that compromise the luminosity.

This work aims to present the state-of-the-art of solutions for indoor location in emergency scenarios. Our research questions are: "What are the requirements for using indoor location systems in emergency situations?"; "Existent indoor location systems support those requirements?"; "There are unattended demands for a new indoor location solution for this domain?".

The paper is organized as follows. Section 2 discusses related work. Section 3 presents the survey protocol. Section 4 reviews and presents a taxonomy of main indoor location techniques. Section 5 discusses the survey results, which include: a requirements list of indoor location systems for emergency scenarios, a summary and analysis of the main works in indoor location for emergency scenarios. Section 6 presents our conclusions and future works.

\section{RELATED WORK}

We identified only one study that performed a review of indoor positioning systems for emergencies scenarios [6]. It 
presents only radio and inertial-based systems. This study does not list the characteristics of emergency situations and does not address new techniques arisen since 2011 .

Other studies performed surveys about location systems in general scenarios [32] and related to specific techniques: radio [35, 32], ultrasonic [14], optical [19] and inertial [12].

In $[28]$ is presented a list of user requirements for location systems in emergency scenarios, extracted from interviews with firefighters, police and military personnel. This work also analysis performance and limitations of GNSS (Global Navigation satellite Systems), radio and inertial navigation systems. In [29] authors present a continuation of the research, bringing a more extensive list of requirements. However, this work does not consider the specifics of each type of emergency scenarios.

\section{A TAXONOMY FOR INDOOR LOCA- TION TECHNIQUES}

Since indoor location literature is vast, comprising different techniques and disciplines, we conducted a preliminary review $^{2}$ before performing the actual survey. The results of this review are presented in Table 1 classified according to: signal type, measurement method and infrastructure needs.

The signal categorization is related to which type of signal is used as reference for the location system. Inertial systems use the direction and acceleration of a moving body to infer its current position. They need to know the body starting position. Optical systems use photos, known markers or lights as reference to positioning. Geomagnetic techniques exploit disturbances on earth magnetic field inside buildings. The disturbances are caused by structural steel elements and they are unique in each building position. Radio techniques compute the radio wave travel time or use the radio signal strength to resolve the tracking. Signals like UWB, ZigBee or WiFi can be used. Similarly, ultrasonic techniques use sound wave travel time as the location system reference.

The measure method is related to how the signal is used to obtain the tracked device position. In angulation the object position is determined identifying the angle at which the signal emitted by the object arrives two or more reference points. The dead-reckoning method is based on analysis of data provided by inertial sensors: knowing the initial position, velocity and direction of the tracked object, one can estimate its current location. In fingerprinting the target area is divided on a grid and signals at each grid cell are captured, forming a map of signals. This map is used to perform tracking. Lateration identifies the object distance to at least three reference points to determine the object position. Proximity determines the location by detecting the presence of the tracked device in one or more sensors neighborhood. Scene analysis involves analysis of the interest location, looking for the tracked device or features that may give a clue to where the viewer is.

Infrastructure categorization examines whether preexisting structure is needed to make the positioning system work. The created category is related to systems that create the location infrastructure on-the-fly. Within the existing category are systems depending on pre-installed infrastrucutre. The no infrastructure category includes systems

${ }^{2} \mathrm{~A}$ more detailed overview about indoor location techniques can be found in http://goo.gl/ULJRJG that works with no infrastructure.

\section{SURVEY PROTOCOL}

This study focuses on indoor location of victims and rescuers inside commercial or residential environments, when emergency situations arise. It seeks to answer the following general research question: "Are there indoor location solutions that meets the requirements of emergency scenarios in indoor environments?". Based on this general issue we seek to answer the following derived questions:

- Q1 What are the requirements for indoor location systems in emergency scenarios?

- Q2 How indoor location systems are positioned to support emergency scenarios?

- Q3 Are there demands for new indoor location solutions for use on emergency scenarios?

\subsection{Search strategy}

The search string used is presented in Table 2. The search was performed in three major electronic databases: Scopus ${ }^{3}$, Compendex ${ }^{4}$ and Web of Science $^{5}$, which encompass the main digital libraries (ACM, IEEE, Elsevier, Springer and Wiley) and includes articles from journals and conferences.

Table 2: Survey search string.

$$
\begin{gathered}
\text { ("indoor" OR "in door" OR "interior" OR "inside" OR } \\
\text { "personnel" OR "person" OR "personal" OR } \\
\text { "pedestrian" OR "in-building" OR "in building" OR } \\
\text { "room" OR "non-gps" OR "non gps") } \\
\text { AND }
\end{gathered}
$$

("positioning" OR "positioner" OR "position" OR "localization" OR "location" OR "locator" OR "locality" OR "geolocation" OR "tracking" OR "navigation" OR "guide" OR "guider" OR "orientation") AND

("emergency" OR "emergencies" OR "crisis" OR "disaster" OR "disasters" OR "rescue" OR "firefighter" OR "firefighters" OR "relief" OR "first responders" OR "first responder" OR "escape" OR "evacuation" OR "danger" OR "dangerous" OR "risk" OR "risky" OR

"hazard" OR "hazardous" OR "unsafe" OR "harmful" OR "critical" OR "distress")

\subsection{Inclusion/exclusion criteria}

The survey considers only primary and secondary studies written in English or Portuguese. The study should present a conceptual or practical implementation of an indoor tracking system facing emergency situations involving buildings evacuation or indoor location of rescuers. The exclusion criteria were applied in the following order:

1. Not a paper: tables, proceedings and non-scientific studies were excluded.

2. Not written in Portuguese or English: used to exclude studies outside the language domain of the survey executors.

\footnotetext{
${ }^{3}$ http://www.scopus.com/home.url

${ }^{4} \mathrm{http}: / /$ www.engineeringvillage.com/

${ }^{5}$ https://sub3.webofknowledge.com
} 
Table 1: Overview of the Taxonomy for Indoor Location Techniques with their advantages/disadvantages.

\begin{tabular}{|c|c|c|}
\hline Category & Advantages & Disadvantages \\
\hline \multicolumn{3}{|c|}{ Signal } \\
\hline Inertial & No infrastructure need & $\begin{array}{l}\text { Needs a reference system to reduce errors and deter- } \\
\text { mine initial position }\end{array}$ \\
\hline Optical & High accuracy & $\begin{array}{l}\text { In most cases is unfeasible to unknown places; requires } \\
\text { high computational power }\end{array}$ \\
\hline Geomagnetism & $\begin{array}{l}\text { Requires only a magnetometer, being accessible for } \\
\text { many devices }\end{array}$ & $\begin{array}{l}\text { Needs mapping the enviroment in a offline phase; Suf- } \\
\text { fers from holes }\end{array}$ \\
\hline Radio & $\begin{array}{l}\text { Can cover large areas; can take advantage of existing } \\
\text { infrastructure; requires small amount of landmarks }\end{array}$ & Suffers from interference caused by objetcts and walls \\
\hline Ultrasonic & High accuracy & $\begin{array}{l}\text { Suffers from interference caused by humidity; requires } \\
\text { high amount of landmarks }\end{array}$ \\
\hline \multicolumn{3}{|c|}{ Measure Method } \\
\hline Angulation & Requires only two reference points & Requires directional antennas \\
\hline Dead-reckoning & Infrastructure independent & $\begin{array}{l}\text { Requires another system to make periodic corrections } \\
\text { and to provide initial position }\end{array}$ \\
\hline Fingerprinting & Robustness & $\begin{array}{l}\text { Unfeasible to unknown places; mapping phase can be } \\
\text { expensive }\end{array}$ \\
\hline Lateration & Position can be estimated based on reference points & Need at least three reference points \\
\hline Proximity & Robustness & Requires high amount of landmarks \\
\hline Scene Analysis & High accuracy & Requires high computional power \\
\hline \multicolumn{3}{|c|}{ Infrastructure } \\
\hline Created & Feasible to unknown places & Needs setup phase \\
\hline Existing & No setup phase & Unfeasible to unknow places \\
\hline No infrastructure & No setup phase; feasible to unknow places & Currently unfeasible \\
\hline
\end{tabular}

3. Has no abstract: studies with no summary were excluded.

4. Duplicated study: studies that appear more than once in the total search result were excluded.

5. It does not cover emergency scenarios in the context of location of victims or rescuers: such as work that deal with emergencies in hospitals, rebuke protests, among others.

6. It does not address conceptual or practical application of indoor location: such as studies focusing on outdoor location systems to collect signals in offline phases, hardware specification for location sensors.

7. It does not address location of humans: works exclusively addressed on robots location were excluded.

8. Unable to get the paper: works whose access was restricted and that could not be obtained even getting in contact with the authors were excluded.

\subsection{Papers selection}

In the first stage of selection (search string execution in databases), 129 papers were obtained. 60 papers were removed by duplicity and 38 papers were removed because they did not fit the study focus, meeting exclusion criterias 5,6 , and 7 . In total, 31 papers were analyzed. Next section discusses results from analyzing those papers in the light of the research questions.

\section{SURVEY RESULTS}

From the 31 papers selected for this survey, 27 papers have functional indoor location systems, 3 papers only describe architecture and do not specify what kind of signal would be used and 1 paper presented a survey about indoor location in emergency scenarios [6].
Furthermore, from 30 studies (architecture or practical application), 23 aimed to support the location of rescuers, 4 instructed victims in an emergency and 1 supported both (victims and rescuers). Two papers did not specify the target user.

Section 5.1 present the list of requirements extracted from this survey (research question Q1). Section [sec:ips-emergency] analyzes the solutions (research questions Q2 and Q3).

\subsection{Emergency scenarios requirements}

Many applications of indoor location systems exist, each having its own set of requirements. Currently no technique is suitable for all scenarios. The most common types of user for these situations are the military, police, firefighters and civilians [29].

Although different users have different location system requirements, part of the requirements are common. In [29] authors present a list of requirements common to military, police and firefighters lifted from discussions and interviews with members of these groups. We have identified some gaps in this list of requirements: 1) the degree of importance of each requirement is not presented; 2) requirements are not facing location systems that meet the victims of emergencies. To fill this gap we performed two actions: review the requirements list proposed by [29] with information collected from the survey papers; and apply a questionnaire with emergency experts to evaluate the new requirements list.

To enhance the requirements list, we extracted the requirements identified by the authors of each paper. Table 3 shows the new requirements list. We classified the requirements according to seven categories:

- Performance: accuracy, precision and speed requirements.

- Robustness: resistance to adverse conditions, battery time and availability. 
- Volume and weight: physical measurements of the carried device(s).

- Usability: ease of use, learning and setup.

- Interface: requirements related to user interface aspects.

- Security: requirements related to security aspects.

- Others: requirements that do not fall into the previous categories.

From this table we can to observe conflicts with some requirements, extracted from distinct works (P1, P2 and P4; $\mathrm{R} 8$ and $\mathrm{R} 9$; V2, V3 and V4). Some requirements are precisely specified (P2, P3, P6, R8, R9, R15, V1, V2, V3, V4, $\mathrm{U} 8$ and $\mathrm{O} 6)$ while others abstract (P1, P5, R10, V5, V6 and O7). Requirement R12 seems to be significant when considering an inertial based location system, given that only this system category is sensitive to movement types. Similarly, U1 is significant considering systems that use created infrastructure. I1, S1 and S2 are applicable to scenarios where armed operational forces are tracked. Requirement U8 was extracted from a paper that presents a system geared to use in urban emergency in Korea. The author explains that in this environment there are small gaps between buildings. Only the front side and indoor environment of the building can be used for the indoor positioning system, which limits the number and position of base stations outside the building.

We decided to conduct a questionnaire directed to specialists in emergencies, with the aim of: revise/update the list of requirements provided in [29]; identify the degree of importance of each requirement of this list and of Table 3; and produce also a list of requirements focused on evacuation of victims of emergency situations. The complete list consisting of the requirements of [29] plus the requirements extracted from this survey papers (Table 3 ) was consolidated and used to compose an online questionnaire, which asked the participant to sort the list as the priority, considering a indoor location system for victims and other one for rescuers.

\subsection{Analyzing indoor location systems for emergency scenarios}

Table 4 presents an overview of the indoor location systems analyzed in this survey, sorted by year in descending order. It shows that there is a predominance of work facing rescuers and systems that use radio signals, especially for the use of RFID. The most adopted measure method is lateration. You may notice an increase in the volume of work per year, demonstrating the interest of the community area, and a larger amount of hybrid system in more recent studies. Oddly, many studies do not report the accuracy obtained. It is a crucial information for the analysis of the proposed location system. Another important information omitted is the precision, reported by only 2 works.

The main conclusions achieved from the analyzed works are:

1. Most works use radio signals for indoor location systems, with emphasis on Ultra Wide Band (UWB). The main reason for adopting this technology is the ability to create infrastructure at the time the emergency occurs, using base stations present in cars or carried by rescuers..

2. There is a predominant focus on rescuers as users for the tracking systems, with space for proposals to locate victims and provide escape routes, especially using smartphones.

3. The majority of published studies present systems for use by firefighters. Only one selected work had focused on military/police.

4. No analyzed work is being used in real situations, because all failed to meet a minimum set of requirements to emergency situations.

5. The studies use different methodologies to evaluate the accuracy/precision of the proposed systems, so that is difficult to compare the performance (such as accuracy and precision) between them. The papers do not detail the experiments whereby the reported accuracies were obtained (many even present precision data). Researches to define test standard methodologies for tracking systems and results documentation are needed.

6. Works using Bluetooth to provide indoor location in emergency scenarios were not found. With the recent emergence of Bluetooth Low Energy (BLE) [9], and marketing of smartphones and tablets supporting this protocol, there is a tendency that this technology becomes common in providing context information such as location.

7. Currently there is no location technology that is best suited for all types of indoor environments. There is a strong tendency for commercial and residential buildings incorporate different indoor location systems to provide context-sensitive information. This creates a heterogeneous scenario where definition of protocols and services that enable both the rescuers and victims use different infrastructure or location information becomes relevant.

\section{CONCLUSIONS AND FUTURE WORK}

This work presented a survey of indoor location systems focused on emergency situations. First related works were presented, highlighting a similar survey [6]. Thus, we detailed the research protocol. The main indoor location techniques were presented, categorized by used signal, measure method and infrastructure need. The contributions of this survey are: a a list of requirements for indoor location systems in emergency scenarios; a list of indoor location systems, classified according to used signal, protocol, infrastructure exigency, analysis method, target user and reported accuracy.

The requirements were used to compose a questionnaire that will be applied to specialists in emergencies. Future work includes finalize and report the results of experts questionnaire, analyze the requirements identified and relate them with the works included in this survey.

As stated in Section 5.2, other interesting future works possibilities are: the proposal for standardized test methodologies for indoor location system; the development of co- 
Table 3: Requirements for Indoor Positioning Systems applied to Emergency Situations. Legend: "User" column: S=Rescuer, V=Victim; "From" column indicates the paper that presents the requirement.

\begin{tabular}{|c|c|c|c|}
\hline $\mathbf{N}$ & Requirement & User & From \\
\hline \multicolumn{4}{|c|}{ Performance } \\
\hline P1 & Horizontal accuracy of about $1 \mathrm{~m}$. & $\mathrm{S}$ & {$[27,6,2$} \\
\hline $\mathrm{P} 2$ & Horizontal accuracy $<1 \mathrm{~m}$. & $\mathrm{S}$ & {$[11,4]$} \\
\hline P3 & Vertical accuracy $<2 \mathrm{~m}$ & $\mathrm{~S}$ & {$[11]$} \\
\hline $\mathrm{P} 4$ & Room level accuracy. & $\mathrm{S}$ & {$[18,10]$} \\
\hline P5 & Speed of calculating and presenting location information. & $\mathrm{S}$ & {$[18$} \\
\hline P6 & Speed of calculating and presenting location information $>1 \mathrm{~Hz}$. & $\mathrm{S}$ & 2 \\
\hline P7 & Location and tracking in 3 dimensions. & $\mathrm{S}$ & 4 \\
\hline \multicolumn{4}{|c|}{ Robustness } \\
\hline R1 & Resistance to heat. & $\mathrm{S}$ & {$[18,25]$} \\
\hline $\mathrm{R} 2$ & Resistance to humidity. & $\mathrm{S}$ & {$[2]$} \\
\hline R3 & Resistance to smoke. & $\mathrm{S}$ & {$[18,2]$} \\
\hline $\mathrm{R} 4$ & Resistance to fire. & $\mathrm{S}$ & {$[2]$} \\
\hline $\mathrm{R} 5$ & Resistance to water. & $\mathrm{S}$ & {$[18$} \\
\hline $\mathrm{R} 6$ & Resistance to NLOS. & $\mathrm{S}$ & {$[2]$} \\
\hline $\mathrm{R} 7$ & Resistance to physical damages. & $\mathrm{S}$ & {$[18,11]$} \\
\hline $\mathrm{R} 8$ & Battery should last for at least 4 hours. & $\mathrm{S}$ & [31] \\
\hline $\mathrm{R} 9$ & Battery should last for at least 24 hours. & $\mathrm{S}$ & {$[11$} \\
\hline R10 & Should be power efficient. & $\mathrm{S}$ & {$[27]$} \\
\hline R11 & Constant accessibility for those who need the positioning data. & $\mathrm{S}$ & [1] \\
\hline $\mathrm{R} 12$ & Should be adaptable to environment change (eg: walls colapse). & $\mathrm{S}$ & {$[6,2]$} \\
\hline $\mathrm{R} 13$ & Must work with different movement types (eg: climb, walk, crouch, etc.). & $\mathrm{S}$ & [27] \\
\hline $\mathrm{R} 14$ & Should work on all building types. & $\mathrm{S}$ & 6 \\
\hline $\mathrm{R} 15$ & Must be able to track at least 100 users simultaneously. & $\mathrm{S}$ & [4] \\
\hline $\mathrm{R} 16$ & Automatic estimation of localization errors (uncertainty). & $\mathrm{S}$ & {$[11,25]$} \\
\hline $\mathrm{R} 17$ & Distance to reference nodes: $100-500 \mathrm{~m}$ & $\mathrm{~S}$ & {$[2]$} \\
\hline \multicolumn{4}{|c|}{ Volume and weight } \\
\hline V1 & Carried device volume should be less then $107 \mathrm{~cm}^{3}$. & $\mathrm{S}$ & [18] \\
\hline$\nabla 2$ & The weight of a node should not exceed 100g. & $\mathrm{S}$ & [31] \\
\hline$\nabla 3$ & The weight of the tracked device should not exceed 1000g. & $\mathrm{S}$ & 11 \\
\hline$\nabla 4$ & The weight of the tracked device should not exceed 1150g. & $\mathrm{S}$ & {$[18$} \\
\hline V5 & Volume should be small. & $\mathrm{S}$ & {$[11,27$} \\
\hline$\nabla 6$ & Weight should be small. & $\mathrm{S}$ & {$[27$} \\
\hline V7 & Should be fit into first responder backpacks. & $\mathrm{S}$ & {$[18]$} \\
\hline \multicolumn{4}{|c|}{ Usability } \\
\hline U1 & Should be easy and fast to setup (created infrastructure). & $\mathrm{S}$ & {$[18]$} \\
\hline $\mathrm{U} 2$ & Sould support a heterogeneous WSN with mobile nodes. & S (firefighter) & 31 \\
\hline U3 & Should be easy to use when wearing fireman gloves. & $\mathrm{S}$ & 31 \\
\hline $\mathrm{U} 4$ & Must be able to do SLAM. & $\mathrm{S}$ & {$[11$} \\
\hline U5 & Should not require site-specif training. & $\mathrm{S}$ & {$[6]$} \\
\hline U6 & Should not require pre-installation. & $\mathrm{S}$ & {$[11,27]$} \\
\hline U7 & Device should be exible and configurable. & S (firefighter) & [31] \\
\hline U8 & Should work with a maximum of 2 base stations, placed in front of the buiding. & $\mathrm{S}$ (firefighter) & [20] \\
\hline U9 & Should work in unknow buildings. & $\mathrm{S}$ & 2 \\
\hline U10 & Should work in buildings with no communication infrastructure. & $\mathrm{S}$ & 2 \\
\hline \multicolumn{4}{|c|}{$\begin{array}{ll}\text { Interface } & \text { Inte }\end{array}$} \\
\hline I1 & Must display where the user is facing. & $\mathrm{S}$ & [11] \\
\hline $\mathrm{I} 2$ & Should notify users when a emergency occurs. & V & {$[16]$} \\
\hline I3 & Should provide emergency exit guidance. & $\mathrm{S}$ & {$[4]$} \\
\hline \multicolumn{4}{|c|}{ Security } \\
\hline S1 & Detection and warning in case of electronic attack. & $\mathrm{S}$ & {$[11]$} \\
\hline S2 & Encrypted voice communications and data transfer. & $\mathrm{S}$ & {$[11]$} \\
\hline \multicolumn{4}{|c|}{$\mathrm{JP}_{\mathrm{T}}$} \\
\hline O1 & Must store information that allows assessment of the operation. & S (firefighter) & [31] \\
\hline $\mathrm{O} 2$ & Must store information that allows improvement of future operations. & S (firefighter) & [31] \\
\hline $\mathrm{O} 3$ & $\begin{array}{l}\text { Data format compatible to and integrated with other information, in particular personal } \\
\text { health status. }\end{array}$ & $\mathrm{S}$ & {$[11,4]$} \\
\hline $\mathrm{O} 4$ & The system should be modular. & $\mathrm{S}$ & [11] \\
\hline $\mathrm{O5}$ & Restricted to equipment that is brought on-site by the relief units themselves. & $\mathrm{S}$ & {$[6]$} \\
\hline $\mathrm{O} 6$ & Cost: <1000 [EUR]. & $\mathrm{S}$ & [11] \\
\hline $\mathrm{O} 7$ & Should not be expensive. & $\mathrm{S}$ & {$[27,6]$} \\
\hline
\end{tabular}


munication protocols for hybrid and ubiquitous location systems; and the research of indoor location systems based on Bluetooth Low Energy.

\section{References}

[1] A. Amanatiadis, A. Gasteratos, and D. Koulouriotis. "An intelligent multi-sensor system for first responder indoor navigation". In: Measurement Science and Technology 22.11 (2011).

[2] S. Beauregard. "Omnidirectional pedestrian navigation for first responders". In: 4th Workshop on Positioning, Navigation and Communication 200\%, WPNC'07 - Workshop Proceedings. Hannover, Germany, 2007, pp. 33-36.

[3] D.M. Do, M.H. Hyun, and Y.B. Choi. "RFID-based indoor location recognition system for emergency rescue evacuation support". In: Lecture Notes in Computer Science (including subseries Lecture Notes in Artificial Intelligence and Lecture Notes in Bioinformatics). Vol. 7861 LNCS. Seoul, Korea, Republic of, 2013, pp. 899-906.

[4] J. Duckworth et al. "WPI precision personnel locator system - Evaluation by first responders". In: 20th International Technical Meeting of the Satellite Division of The Institute of Navigation 2007 ION GNSS $200 \%$. Vol. 5. Fort Worth, TX, United states, 2007, pp. 14271435.

[5] C. El Amine and O. Mohammed. "A localization and an identification system of personnel in areas at risk using a wireless sensor network". In: Konya, Turkey, 2013, pp. 127-131.

[6] C Fuchsa et al. "Indoor tracking for mission critical scenarios: A survey". In: Pervasive and Mobile Computing (Feb. 2011), pp. 1-14.

[7] R. Giuliano et al. "Indoor localization system for first responders in emergency scenario". In: 2013 9th International Wireless Communications and Mobile Computing Conference, IWCMC 2013. Cagliari, Sardinia, Italy, 2013, pp. 1821-1826.

[8] G. Glanzer. "Personal and first-responder positioning: State of the art and future trends". In: 2012 Ubiquitous Positioning, Indoor Navigation, and Location Based Service, UPINLBS 2012. 2012.

[9] Bluetooth Special Interest Group. Bluetooth. July 2014.

[10] J. R. Guerrieri et al. "RFID-assisted indoor localization and communication for first responders". In: $E u$ ropean Space Agency, (Special Publication) ESA SP. Vol. 626 SP. Nice, France, 2006.

[11] K. V. S. Hari et al. "A prototype of a first-responder indoor localization system". In: Journal of the Indian Institute of Science 93.3 (2013), pp. 511-520.

[12] R. Harle. "A Survey of Indoor Inertial Positioning Systems for Pedestrians". In: IEEE Communications Surveys and Tutorials (Jan. 2013), pp. 1281-1293.

[13] D. Harmer et al. "A ultra-wide band indoor personnel tracking system for emergency situations (europcom)". In: 2008 5th European Radar Conference Proceedings, EuRAD 2008. Amsterdam, Netherlands, 2008, pp. 404-407.
[14] F. Ijaz et al. "Indoor positioning: A review of indoor ultrasonic positioning systems". In: 2013 15th International Conference on Advanced Communication Technology (ICACT) (Jan. 2013), pp. 1146-1150.

[15] S. J. Ingram, D. Harmer, and M. Quinlan. "Ultra wide band indoor positioning systems and their use in emergencies". In: Record - IEEE PLANS, Position Location and Navigation Symposium. Monterey, CA, United states, 2004, pp. 706-715.

[16] Y. Inoue et al. "Indoor emergency evacuation service on autonomous navigation system using mobile phone". In: Proceedings of the 2nd International Symposium on Universal Communication, ISUC 2008. Osaka, Japan, 2008, pp. 79-85.

[17] E. Kaplan and J. Hegarty. Understanding GPS: Principles and Applications. Second. Artech House mobile communications series. Artech House, 2005, p. 723. ISBN: 9781580538954.

[18] Nan Li et al. "A BIM centered indoor localization algorithm to support building fire emergency response operations". In: Automation in Construction 42 (2014), pp. $78-89$.

[19] R. Mautz and S. Tilch. "Survey of optical indoor positioning systems". In: 2011 International Conference on Indoor Positioning and Indoor Navigation (IPIN) (Sept. 2011), pp. 1-7.

[20] G. B. Moon, M. Beom, and G. Jee. "An indoor positioning system for a first responder in an emergency environment". In: International Conference on Control, Automation and Systems. Jeju, Korea, Republic of, 2012, pp. 1368-1372.

[21] G. B. Moon et al. "A robust indoor positioning system using two-stage EKF SLAM for first responders in an emergency environment". In: International Conference on Control, Automation and Systems. Gwangju, Korea, Republic of, 2013, pp. 707-711.

[22] B.S. Nie et al. "Internet of things-based positioning of coalmine personnel and monitoring of emergency state". In: Proceedings of the 2011 2nd International Conference on Digital Manufacturing and Automation, ICDMA 2011. Zhangjiajie, Hunan, China, 2011, pp. 657-660.

[23] Y. Noh et al. "CLIPS: Infrastructure-free collaborative indoor positioning scheme for time-critical team operations". In: 2013 IEEE International Conference on Pervasive Computing and Communications, PerCom 2013. San Diego, CA, United states, 2013, pp. 172178.

[24] Lauro Ojeda and Johann Borenstein. "Non-GPS Navigation for Security Personnel and First Responders". In: Journal of Navigation 60 (03 Sept. 2007), pp. 391407. DOI: $10.1017 / \mathrm{S} 0373463307004286$.

[25] F Pascucci and R Setola. "An indoor localization framework for hybrid rescue teams". In: IFAC Proceedings Volumes (IFAC-PapersOnline). Vol. 18. PART 1. Milano, Italy, 2011, pp. 4765-4770. 
Table 4: Evaluation of the Indoor Positioning Systems. Legend: "Signal" column, R=Radio, I=Inertial, $\mathrm{M}=$ Geomagnetism, $\mathrm{O}=$ Optical; "Inf. (Infrastructure)" column: $\mathrm{E}=$ Exist, $\mathrm{C}=$ Created, N=None; "U (User)" column: $\mathrm{S}=$ Rescuer, $\mathrm{V}=$ Victim; In all columns: ?=Not informed, $*=\mathrm{All}$, $-=$ Not apply.

\begin{tabular}{|c|c|c|c|c|c|c|c|}
\hline Ref & Year & Signal & Protocol & In & Analysis Method & $\mathrm{U}$ & Accuracy \\
\hline 18 & 2014 & $\mathrm{R}$ & * & $\mathrm{C}$ & Lateration (RSSI) & $\mathrm{S}$ & $<=2.02 \mathrm{~m}$ \\
\hline 5$]$ & 2013 & $\mathrm{R} / \mathrm{I}$ & 802. $15.4+$ RFID & $\mathrm{E}$ & Lateration(RSSI)/ Proximidity & V & $<=1.7$ \\
\hline [11] & 2013 & $\mathrm{I}$ & - & $\mathrm{N}$ & Inertial (ZUPT) & $\mathrm{S}$ & $<=2 \mathrm{~m}$ \\
\hline$[21]$ & 2013 & $\mathrm{R}$ & 802.15 .4 & $\mathrm{C}$ & Lateration (TOA + SLAM) & $\mathrm{S}$ & $<=2 \mathrm{~m}$ \\
\hline 23 & 2013 & $\mathrm{R} / \mathrm{I}$ & 802.11 & $\mathrm{~N}$ & Lateration (RSSI)/DR & $\mathrm{S}$ & $?$ \\
\hline 7$]$ & 2013 & $\mathrm{R}$ & RFID & $\mathrm{E}$ & Proximidity & $\mathrm{S}$ & ? \\
\hline$[37]$ & 2013 & $\mathrm{M} / \mathrm{I}$ & - & $\mathrm{N}$ & DR (ZUPT) & $\mathrm{S}$ & $2,60 \mathrm{~m}$, environments with stairs \\
\hline$[3]$ & 2013 & $\mathrm{R}$ & RFID & $\mathrm{E}$ & Lateration (RSSI) & $\mathrm{V}$ & $168 \mathrm{~cm}$ average \\
\hline$[36]$ & 2013 & $\mathrm{R}$ & UWB & $\mathrm{C}$ & Lateration (TOA) & $\mathrm{S}$ & From $0.03 \mathrm{~m}$ to unfeasible \\
\hline 26 & 2012 & $\mathrm{R} / \mathrm{I}$ & $*$ & & - & $\mathrm{S}$ & - \\
\hline$[8]$ & 2012 & I & - & $\mathrm{N}$ & DR (ZUPT) & $\mathrm{S}$ & - \\
\hline$[40]$ & 2012 & $\mathrm{R}$ & $?$ & $\mathrm{E}$ & Lateration (TDOA) & $\mathrm{S}$ & $?$ \\
\hline 27 & 2011 & $\mathrm{R} / \mathrm{I}$ & UWB & $\mathrm{N}$ & Inertial (ZUPT) & $\mathrm{S}$ & $3.5 \mathrm{~m}$ in a $194 \mathrm{~m}$ route \\
\hline 25 & 2011 & $\mathrm{R}$ & RFID & $\mathrm{E}$ & Lateration (RSSI) & $\mathrm{S}$ & $?$ \\
\hline$[1]$ & 2011 & $\mathrm{R} / \mathrm{O} / \mathrm{I}$ & RFID & $\mathrm{E}$ & Fingerprinting (Wifi)/DR & $\mathrm{S}$ & Between $2.58 \mathrm{~m}$ and $19.9 \mathrm{~m}$ \\
\hline 6 & 2011 & - & - & - & - & - & 5 \\
\hline$[22]$ & 2011 & $\mathrm{R}$ & ZigBee & $\mathrm{E}$ & Lateration (RSSI) & V & $?$ \\
\hline 31 & 2010 & $\mathrm{R}$ & 802.15 .4 & $\mathrm{C}$ & ? & S & $?$ \\
\hline$[38]$ & 2010 & $\mathrm{R}$ & 802.11 & $\mathrm{E}$ & Lateration (RSSI) & $?$ & Between $68.9 \%$ and $80.6 \%$. \\
\hline [13] & 2008 & $\mathrm{R}$ & UWB & $\mathrm{C}$ & Lateration (TOA) & $\mathrm{S}$ & $<=1 \mathrm{~m}$ \\
\hline 34 & 2008 & $\mathrm{R}$ & UWB & $\mathrm{C}$ & Lateration (TOA) & $\mathrm{S}$ & $?$ \\
\hline 30 & 2008 & $\mathrm{R}$ & RFID, UWB, WiFi & $\mathrm{E}$ & Proximity, Lateration and Fingerprint & $\mathrm{S}$ & $?$ \\
\hline$[16]$ & 2008 & $\mathrm{R}$ & VHF & $\mathrm{E}$ & ? & $\mathrm{V}$ & $?$ \\
\hline 41 & 2008 & $\mathrm{R}$ & RFID & $\mathrm{E}$ & Proximidity & $?$ & $?$ \\
\hline 24$]$ & 2007 & I & - & $\mathrm{N}$ & DR (ZUPT) & $\mathrm{S}$ & $98 \%$ in 15 minutes routes. \\
\hline 2 & 2007 & I & - & $\mathrm{N}$ & DR (ZUPT) & $\mathrm{S}$ & ? \\
\hline 4 & 2007 & $\mathrm{R}$ & $?$ & $\mathrm{C}$ & Lateration (TDOA) & $\mathrm{S}$ & $<1 \mathrm{~m}$ \\
\hline$[10]$ & 2006 & $\mathrm{R} / \mathrm{I}$ & RFID & $\mathrm{E}$ & Proximidity & $\mathrm{S}$ & - \\
\hline 33 & 2005 & $\mathrm{R}$ & 802.11 & $\mathrm{E}$ & Lateration (TOA) & $\mathrm{S}$ & $<=6 \mathrm{~m}$ \\
\hline 15$]$ & 2004 & $\mathrm{R}$ & UWB & $\mathrm{E}$ & Lateration (TDOA) & $*$ & $?$ \\
\hline
\end{tabular}

[26] F. Pascucci et al. "A REference implementation of interoperable indoor location communication systems for First REsponders: The REFIRE project". In: 2012 IEEE International Symposium on Safety, Security, and Rescue Robotics, SSRR 2012. College Station, TX, United states, 2012.

[27] J. Rantakokko et al. "Accurate and reliable soldier and first responder indoor positioning: Multisensor systems and cooperative localization". In: IEEE Wireless Communications 18.2 (2011), pp. 10-18.

[28] J. Rantakokko et al. Positioning of emergency personnel in rescue operations - possibilities and vulnerabilities with existing techniques and identification of needs for future RD. Tech. rep. Swedish Defence Research Agency, Royal Institute of Technology, Luleå University of Technology, Swedish Defence Materiel Administration, SAAB Bofors Dynamics AB, SAAB Aerotech AB, Swedish Rescue Services Agency, Rescue Service in Linkoping, National Criminal Police, Army Combat School, 2007.

[29] J. Rantakokko et al. "User requirements for localization and tracking technology: A survey of missionspecific needs and constraints". In: International Conference on Indoor Positioning and Indoor Navigation (IPIN) (Sept. 2010), pp. 1-9.

[30] U. Rueppel and K. Stuebbe. "BIM-Based IndoorEmergency-Navigation-System for Complex Buildings". In: Tsinghua Science and Technology 13.SUPPL. 1 (2008), pp. 362-367.

[31] M. Scholz, T. Riedel, and C. Decker. "A flexible architecture for a robust indoor navigation support device for firefighters". In: INSS 2010 - 7th International Conference on Networked Sensing Systems. Kassel, Germany, 2010, pp. 227-232.

[32] J. Torres-Solis, T. Falk, and T. Chau. "A Review of Indoor Localization Technologies: towards Navigational Assistance for Topographical Disorientation". In: Ambient Intelligence (Mar. 2010), pp. 51-84.

[33] P. Womble, A. Barzilov, and J. Paschal. "A tracking technology for security personnel and first responders". In: Proceedings of SPIE - The International Society for Optical Engineering. Vol. 5778. PART I. Orlando, FL, United states, 2005, pp. 51-56.

[34] L. Xia et al. "An ultra-wide band based ad hoe networking scheme for personnel tracking in emergencies". In: Proceedings of the 2nd International Conference on Pervasive Computing Technologies for Healthcare 2008, PervasiveHealth. Tampere, Finland, 2008, pp. $78-81$.

[35] J. Xiao et al. "Comparison and Analysis of Indoor Wireless Positioning Techniques". In: 2011 International Conference on Computer Science and Service System (CSSS) (June 2011), pp. 293-296.

[36] L. Zhang et al. "Ultra wideband indoor positioning system in support of emergency evacuation". In: Proceedings of the 5th ACM SIGSPATIAL International Workshop on Indoor Spatial Awareness, ISA 2013. Orlando, FL, United states, 2013, pp. 42-49.

[37] R. Zhang, F. Hoflinger, and L. Reindl. "Inertial sensor based indoor localization and monitoring system for emergency responders". In: IEEE Sensors Journal 13.2 (2013), pp. 838-848. 
[38] M. Zhao et al. "Directional Wi-Fi based indoor location system for emergency". In: Proceedings - Symposia and Workshops on Ubiquitous, Autonomic and Trusted Computing in Conjunction with the UIC 2010 and ATC 2010 Conferences, UIC-ATC 2010. Xi'an, China, 2010, pp. 501-502.

[39] D. Zhongliang et al. "Situation and Development Tendency of Indoor Positioning". In: China Communications (Mar. 2013), pp. 42-55.

[40] X. Zhou, H. Zhang, and L. Sun. "Research on Location Technology in Building Fire Rescue". In: CONFERENCE ON MODELING, IDENTIFICATION AND CONTROL. Ed. by W Deng. Vol. 3. AASRI Procedia. 2012, pp. 445-450. DOI: 10.1016/j.aasri. 2012. 11.070 .

[41] B. Zoghi and B. McKee. "RFID-based network for personnel and mission-critical asset tracking in a Disaster City\&reg". In: Proceedings - Frontiers in Education Conference, FIE. Saratoga Springs, NY, United states, 2008, S2C7 -S2C12. 